\title{
Elastic Labels on the Perimeter of a Rectangle
}

\author{
Claudia Iturriaga and Anna Lubiw * \\ Dept. of Computer Science, University of Waterloo \\ cciturri@daisy.uwaterloo.ca \\ alubiw@daisy. uwaterloo.ca
}

An important and challenging task in cartography is the labeling of mapsattaching text to geographic features. Usually, the features to be labeled are regions, lines, and points on a map. One of the traditional formulations of the problem of labeling points is the point-feature-label placement problem, where we are given a set of points in the plane, and an axis-parallel rectangular label associated with each point, and the problem is to place each label with one corner at its associated point such that no two labels overlap. KIm88, FWa91, MSh91, KRa92 This problem is known to be NP-complete. There are approximation algorithms when the problem consists of scaling the labels.

In this paper we propose an alternative where each label is a rectangle with fixed area, but varying height and width. The elastic labeling problem is to choose the height and width of each label, and the corner of the label to place at the associated point, so that no two labels overlap.

The elastic labeling problem is useful when the goal of placing a label at a given point is to associate with the point some text consisting of more than one word. In this case we can write the specified text inside the label using one, two, or more rows, as long as the label is placed at the specified point.

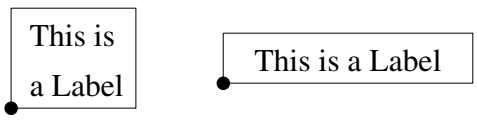

Fig. 1. An Elastic Label.

Our main result is a polynomial time algorithm for the special case of the elastic labeling problem when the given points lie on the boundary of our rectangular map. This "rectangle perimeter labeling problem" arises when the perimeter of the map is labeled with information about objects that lie beyond the boundary of the map, e.g. where the roads lead to, etc. This problem is likely to be relevant in GIS as maps are displayed dynamically on a computer screen using clipping, panning, and zooming.

To solve the rectangle perimeter labeling problem, we first tackle two subproblems where the points lie on only two sides of the rectangle, either two adjacent sides or two opposite sides. We call the case where the points lie on two

* Research partially supported by NSERC 


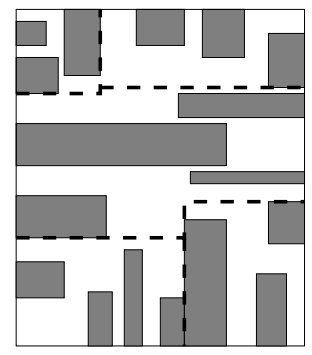

Fig. 2. A decomposition.

adjacent sides of the rectangle the two-axis labeling problem, and the case where the points lie on two opposite sides of the rectangle the two-parallel lines labeling problem. Our polynomial time algorithm for the two-axis labeling problem was presented in ILu97b.

Here, we present a polynomial time algorithm for the two-parallel lines labeling problem, and we show how to solve the rectangle perimeter labeling problem in polynomial time using algorithms for the two subproblems.

For the two-parallel lines labeling problem we use a greedy approach, adding elastic rectangles from bottom to top, keeping the most recently added rectangles elastic, and fixing the earlier ones. To solve the rectangle perimeter labeling problem, we show that for any instance of the problem it suffices to consider a polynomial number of decompositions of the rectangle into regions in which labels from only two sides of the rectangle compete for space. (It is intuitively clear that near a corner of the map, for example, only the labels from two sides of the map are relevant.) Thus we can apply our algorithms for the special cases in these regions. The dashed lines in Figure 2 illustrate one such decomposition.

\section{References}

[FWa91] M. Formann and F. Wagner. A packing problem with applications in lettering of maps. Proceedings of the 7th ACM Symposium on Computational Geometry. (1991) 281-288.

[KIm88] T. Kato and H. Imai. The NP-completeness of the character placement problem of 2 or 3 degrees of freedom. Record of Joint Conference of Electrical and Electronic engineers in Kyushu. (1988), 1138. In Japanese.

[KRa92] D. Knuth and A. Raghunathan. The problem of compatible representatives. SIAM Disc. Math. (1992) 5 (3), 422-427.

[ILu97a] C. Iturriaga and A. Lubiw. NP-hardness of some map labeling problems. Technical Report CS-97-18. University of Waterloo (1997).

[ILu97b] C. Iturriaga and A. Lubiw. Elastic labels: the two-axis case. In G. Di Battista editor, Graph Drawing (Proc. GD'97). vol. 1353 of Lecture Notes in Computer Science. Springer-Verlag. (1998), 181-192.

[MSh91] J. Marks and S. Shieber. The computational complexity of cartographic label placement. Technical Report CRCT-05-91. Harvard University (1991). 